\title{
Infinitely many solutions for a fourth-order Kirchhoff type elliptic problem
}

\author{
M. Massar ${ }^{1}$, E. M. Hssini ${ }^{1}$, N. Tsouli ${ }^{1}$, M. Talbi $^{2}$ \\ ${ }^{1}$ Department of Mathematics, University Mohamed I, Oujda, Morocco \\ ${ }^{2}$ CRMEF, Oujda, Morocco
}

\section{massarmed@hotmail.com}

Article history:

Received May 2013

Accepted June 2013

Available online June 2013

\section{Abstract}

This paper studies the existence of infinitely many solutions for a fourth-order Kirchhoff type elliptic problem

$$
\begin{cases}\Delta\left(|\Delta u|^{p-2} \Delta u\right)-\left[M\left(\int_{\Omega}|\nabla u|^{p} d x\right)\right]^{p-1} \Delta_{p} u+\rho|u|^{p-2} u=\lambda f(x, u) & \text { in } \Omega \\ u=\Delta u=0 & \text { on } \partial \Omega .\end{cases}
$$

Our technical approach is based on Ricceri's principle variational.

Keywords: Navier boundary, nonlocal, Ricceri's variational principle.

\section{Introduction}

In this article, we are concerned with the existence of infinitely many solutions for a fourth-order $p$ Kirchhoff elliptic problem

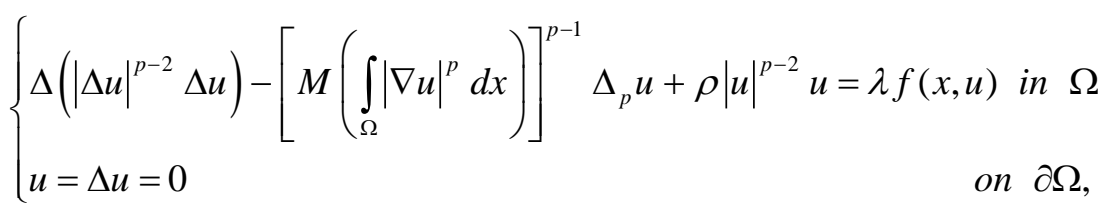


where $p>\max \left\{1, \frac{N}{2}\right\}, \Omega \subset \mathbb{R}^{N}(N \geq 1)$ is a bounded smooth domain, $\lambda, \rho>0$. The perturbation

$f: \bar{\Omega} \times \mathbb{R} \rightarrow \mathbb{R}$ is a continuous function and $M: \mathbb{R}^{+} \rightarrow \mathbb{R}$ is a continuous function with the following bounded condition:

(M) There are two positive constants $m_{0}, m_{1}$ such that

$$
m_{0} \leq M(t) \leq m_{1} \text {, for all } t \geq 0
$$

The problem (1.1) is related to the stationary problem of a model introduced by Kirchhoff [15]. More precisely, Kirchhoff introduced a model given by the following equation

$$
\rho \frac{\partial^{2} u}{\partial t^{2}}-\left(\frac{\rho_{0}}{h}+\frac{E}{2 L} \int_{0}^{L}\left|\frac{\partial u}{\partial x}\right|^{2} d x\right) \frac{\partial^{2} u}{\partial x^{2}}=0,
$$

which extends the classical D'Alembert's wave equation by considering the effects of the changes in the length of the strings during the vibrations. Latter (1.3) was developed to form

$$
u_{t t}-M\left(\int_{\Omega}|\nabla u|^{2} d x\right) \Delta u=f(x, u) \text { in } \Omega .
$$

After that, many authors studied the following nonlocal elliptic boundary value problem

$$
-M\left(\int_{\Omega}|\nabla u|^{2} d x\right) \Delta u=f(x, u) \text { in } \Omega, u=0 \text { on } \partial \Omega .
$$

Problems like (1.5) can be used for modeling several physical and biological systems where $u$ describes a process which depends on the average of it self, such as the population density, see [3]. Many interesting results for problems of Kirchhoff type were obtained see for example $[3,10,11,12,13,16$, 21]. The investigation of fourth order boundary value problems has drawn the attention of many authors, because the static form change of beam or the sport of rigid body can be described by a fourth order equation, and specially a model to study travelling waves in suspension bridges can be furnished by the fourth order equation of nonlinearity. Several results are known concerning the existence and multiplicity of solutions for fourth order boundary value problems, see $[1,4,5,6,8,9,17]$ and the references therein. In [21], wang and An studied the following fourth-order nonlocal elliptic problem

$$
\begin{cases}\Delta^{2} u-M\left(\int_{\Omega}|\nabla u|^{2} d x\right) \Delta u=\lambda f(x, u) & \text { in } \Omega \\ u=\Delta u=0 & \text { on } \partial \Omega .\end{cases}
$$

By using the mountain pass theorem, the authors established the existence and multiplicity of solutions.

Motivated by the paper [9], we look for the existence of infinitely many solutions of problem (1.1) More precisely, we will prove the existence of well precise intervals of parameters such that problem (1.1) admits either an unbounded sequence of solutions provided that the functions $f(x, u)$ and $M$ has 
a suitable behaviour at infinity or a sequence of nontrivial solutions converging to zero if a similar behaviour occurs at zero. Our main tool is a general critical points theorem due to Bonanno and Molica Bisci [7] that is a generalization of a previous result of Ricceri [19].

In the sequel, $X$ denotes the space $W^{2, p}(\Omega) \cap W_{0}^{2, p}(\Omega)$, which is a reflexive Banach space endowed with the norm

$$
\|u\|=\left(\int_{\Omega}|\Delta u|^{p} d x+\int_{\Omega}|\nabla u|^{p} d x+\int_{\Omega}|u|^{p} d x\right)^{\frac{1}{p}} .
$$

Let

$$
K:=\sup _{u \in X \backslash\{0\}} \frac{\max _{x \in \bar{\Omega}}|u(x)|^{p}}{\|u\|^{p}} .
$$

Since $p>\max \left\{1, \frac{N}{2}\right\}$, the Rellich Kondrachov theorem assures that $W^{2, p}(\Omega) \cap W_{0}^{2, p}(\Omega) \hookrightarrow C(\bar{\Omega})$ is compact, and hence $K<\infty$.

We say that $u \in X$ is a weak solution of problem (1.1) if

$\int_{\Omega}|\Delta u|^{p-2} \Delta u \Delta v d x+\left[M\left(\int_{\Omega}|\nabla u|^{p} d x\right)\right]_{\Omega}^{p-1} \int_{\Omega}|\nabla u|^{p-2} \nabla u \nabla v d x+\rho \int_{\Omega}|u|^{p-2} u v d x-\lambda \int_{\Omega} f(x, u) v d x=0$,

for all $v \in X$. The existence of solutions understood as critical points of the energy functional $I_{\lambda}: X \rightarrow \mathbb{R}$ given by

$$
I_{\lambda}(u)=\frac{1}{p} \int_{\Omega}|\Delta u|^{p} d x+\frac{1}{p} \widehat{M}\left(\int_{\Omega}|\nabla u|^{p} d x\right)+\frac{\rho}{p} \int_{\Omega}|u|^{p} d x-\lambda \int_{\Omega} F(x, u) d x,
$$

for all $u \in X$, where

$$
\widehat{M}(t)=\int_{0}^{t}[M(s)]^{p-1} d s \quad \text { and } \quad F(x, t)=\int_{0}^{t} f(x, s) d s
$$

Put

$$
M^{-}:=\min \left\{1, m_{0}^{p-1}, \rho\right\}
$$

Fix $x^{0} \in \Omega$ and pick $s_{1}>0$ such that $B\left(x^{0}, s_{1}\right) \subset \Omega$, where $B\left(x^{0}, s_{1}\right)$ denotes the ball with center at $x^{0}$ and radius of $s_{1}$. Set 


$$
\begin{gathered}
\theta_{1}=\theta_{1}\left(N, p, s_{1}\right)=\frac{2 \pi^{\frac{N}{2}}}{\Gamma\left(\frac{N}{2}\right)} \int_{\frac{s_{1}}{2}}^{s_{1}} \mid \frac{12(N+1)}{s_{1}^{3}} r-\frac{24 N}{s_{1}^{2}}+\frac{9(N-1)}{s_{1}} \frac{1}{r} r^{N-1} d r, \\
\theta_{2}=\theta_{2}\left(N, p, x^{0}, s_{1}\right)=\frac{2 \pi^{\frac{N}{2}}}{\Gamma\left(\frac{N}{2}\right)} \int_{B\left(x^{0}, s_{1}\right) \backslash B\left(x^{0}, \frac{s_{1}}{2}\right)}\left[\sum_{i=1}^{N}\left(\frac{12\left(x_{I}-x_{i}^{0}\right)}{s_{1}^{3}}-\frac{24\left(x_{I}-x_{i}^{0}\right)}{s_{1}^{2}}+\frac{9\left(x_{I}-x_{i}^{0}\right)}{s_{1} l}\right)^{2}\right]^{\frac{p}{2}} d x, \\
\theta_{3}=\theta_{3}\left(N, p, s_{1}\right)=\frac{2 \pi^{\frac{N}{2}}}{\Gamma\left(\frac{N}{2}\right)}\left(\frac{\left(\frac{s_{1}}{2}\right)^{N}}{N}+\int_{\frac{s_{1}}{2}}^{s_{1}}\left|\frac{4}{s_{1}^{3}} r^{3}-\frac{12}{s_{1}^{2}} r^{2}+\frac{9}{s_{1}} r-1\right| r^{N-1} d r\right),
\end{gathered}
$$

with $l=\operatorname{dist}\left(x, x^{0}\right)=\sqrt{\sum_{i=1}^{N}\left(x_{i}-x_{i}^{0}\right)^{2}}$ and $\Gamma$ denotes the Gamma function, and let

$$
L:=\frac{M^{-}}{K\left(\theta_{1}+m_{1}^{p-1} \theta_{2}+\rho \theta_{3}\right)}
$$

Now we are ready to state our main results.

Theorem 1.1. Assume that (M) holds and

(i. $F(x, t) \geq 0$ for every $(x, t) \in \Omega \times[0,+\infty)$;

$\left(i_{2}\right)$ There exist $x^{0} \in \Omega, s_{1}>0$ as considered in (1.8) such that, if we put

$$
\alpha:=\liminf _{\sigma \rightarrow+\infty} \frac{\int_{\Omega} \sup _{|t| \leq \sigma} F(x, t) d x}{\sigma^{p}}, \quad \beta:=\limsup _{t \rightarrow+\infty} \frac{\int_{B\left(x^{0}, \frac{s_{1}}{2}\right)} F(x, t) d x}{t^{p}},
$$

one has

$$
\alpha<L \beta \text {. }
$$

Then, for every

$$
\left.\lambda \in \Lambda:=\frac{M^{-}}{K p}\right] \frac{1}{L \beta}, \frac{1}{\alpha}[
$$

problem (1.1) admits an unbounded sequence of weak solutions. 
Theorem 1.2. Assume that (M), $\left(i_{1}\right)$ hold and

$\left(i_{3}\right)$ There exist $x^{0} \in \Omega, s_{1}>0$ as considered in (1.8) such that, if we put

$$
\alpha^{0}:=\liminf _{\sigma \rightarrow 0^{+}} \frac{\int_{\Omega} \sup _{|t| \leq \sigma} F(x, t) d x}{\sigma^{p}}, \quad \beta^{0}:=\limsup _{t \rightarrow 0^{+}} \frac{\int_{\left(x^{0}, \frac{s_{1}}{2}\right)} F(x, t) d x}{t^{p}},
$$

one has

$$
\alpha^{0}<L \beta^{0}
$$

Then, for every

$$
\left.\lambda \in \Lambda^{0}:=\frac{M^{-}}{K p}\right] \frac{1}{L \beta^{0}}, \frac{1}{\alpha^{0}}[
$$

problem (1.1) admits a sequence $\left(u_{n}\right)$ of weak solutions such that $u_{n} \rightarrow 0$ strongly.

\section{Proof of main results}

For the reader's convenience, we recall one smooth version of Ricceri's variational principle [19], which is our main tool.

Theorem 2.1. Let $X$ be a reflexive real Banach space, let $\Phi, \Psi: X \rightarrow \mathbb{R}$ be two Gâteaux differentiable functionals such that $\Phi$ is sequentially weakly lower semicontinuous, strongly continuous and coercive and $\Psi$ is sequentially weakly upper semicontinuous. For every $r>\inf _{X} \Phi$, let us put

$$
\varphi(r):=\inf _{u \in \Phi^{-1}(]-\infty, r[)} \frac{\left(\sup _{v \in \Phi^{-1}(]-\infty, r[)} \Psi(v)\right)-\Psi(u)}{r-\Phi(u)}
$$

and

Then, one has

$$
\gamma:=\liminf _{r \rightarrow+\infty} \varphi(r), \quad \delta:=\liminf _{r \rightarrow\left(\inf _{X} \Phi\right)^{+}} \varphi(r) .
$$

(a) for every $r>\inf _{X} \Phi$ and every $\left.\lambda \in\right] 0, \frac{1}{\varphi(r)}\left[\right.$, the restriction of the functional $I_{\lambda}=\Phi-\lambda \Psi$ to $\Phi^{-1}(]-\infty, r[)$ admits a global minimum, which is a critical point (local minimum) of $I_{\lambda}$ in $X$;

(b) if $\gamma<+\infty$ then, for each $\lambda \in] 0, \frac{1}{\gamma}[$, the following alternative holds:

either 
$\left(b_{1}\right) I_{\lambda}$ possesses a global minimum,

or

$\left(b_{2}\right)$ there is a sequence $\left(u_{n}\right)$ of critical points (local minima) of $I_{\lambda}$ such that $\lim _{n \rightarrow+\infty} \Phi\left(u_{n}\right)=+\infty$;

(c) $\delta<+\infty$ then, for each $\lambda \in] 0, \frac{1}{\delta}[$, the following alternative holds:

either

$\left(c_{1}\right)$ there is a global minimum of $\Phi$ which is a local minimum of $I_{\lambda}$,

or

$\left(c_{2}\right)$ there is a sequence of pairwise distinct critical points (local minima) of $I_{\lambda}$ which weakly converges to global minimum of $\Phi$.

Before proving the results, we introduce the functionals

$$
\Phi(u)=\frac{1}{p} \int_{\Omega}|\Delta u|^{p} d x+\frac{1}{p} \widehat{M}\left(\int_{\Omega}|\nabla u|^{p} d x\right)+\frac{\rho}{p} \int_{\Omega}|u|^{p} d x, \quad \Psi(u)=\int_{\Omega} F(x, u) d x .
$$

It is well known that $\Phi$ and $\Psi$ are well defined and Gâteaux differentiable whose Gâteaux derivatives at $u \in X$ are given by

$$
\begin{gathered}
\left\langle\Phi^{\prime}(u), v\right\rangle=\int_{\Omega}|\Delta u|^{p-2} \Delta u \Delta v d x+\left[M\left(\int_{\Omega}|\nabla u|^{p} d x\right)\right] \int_{\Omega}^{p-1}|\nabla u|^{p-2} \nabla u \nabla v d x+\rho \int_{\Omega}|u|^{p-2} u v d x, \\
\left\langle\Psi^{\prime}(u), v\right\rangle=\int_{\Omega} f(x, u) v d x,
\end{gathered}
$$

for all $v \in X$. Moreover $\Phi$ is strongly continuous, since $\Psi$ has a compact derivative, it results sequentially weakly continuous. Also, $\Phi$ is sequentially weakly lower semicontinuous. In fact, let $u_{n} \rightarrow u$ weakly in $X$. By the weakly lower semicontinuity of norm, it follows that

$$
\begin{array}{r}
\int_{\Omega}|\Delta u|^{p} d x \leq \liminf _{n \rightarrow+\infty} \int_{\Omega}\left|\Delta u_{n}\right|^{p} d x, \\
\quad \int_{\Omega}|u|^{p} d x \leq \liminf _{n \rightarrow+\infty} \int_{\Omega}\left|u_{n}\right|^{p} d x, \\
\int_{\Omega}|\nabla u|^{p} d x \leq \liminf _{n \rightarrow+\infty} \int_{\Omega}\left|\nabla u_{n}\right|^{p} d x .
\end{array}
$$


In view of the monotonicity and continuity of $\widehat{M}$, we deduce from (2.3) that

$$
\widehat{M}\left(\int_{\Omega}|\nabla u|^{p} d x\right) \leq \widehat{M}\left(\liminf _{n \rightarrow+\infty} \int_{\Omega}\left|\nabla u_{n}\right|^{p} d x\right) \leq \liminf _{n \rightarrow+\infty} \widehat{M}\left(\int_{\Omega}\left|\nabla u_{n}\right|^{p} d x\right) .
$$

This and (2.2) imply that $\Phi$ is sequentially weakly lower semicontinuous. Now, let us show that $\Phi$ is coercive. By (M), for every $u \in X$, we have

$$
\begin{aligned}
\Phi(u) & \geq \frac{1}{p} \int_{\Omega}|\Delta u|^{p} d x+\frac{m_{0}^{p-1}}{p} \int_{\Omega}|\nabla u|^{p} d x+\frac{\rho}{p} \int_{\Omega}|u|^{p} d x \\
& \geq \frac{M^{-}}{p}\|u\|^{p} .
\end{aligned}
$$

Consequently, $\Phi$ is coercive.

\subsection{Proof of Theorem 1.1.}

Let $\Phi$ and $\Psi$ be defined as in (2.1). Then

$$
I_{\lambda}(u)=\Phi(u)-\lambda \Psi(u), \text { for all } u \in X
$$

In order to apply Theorem 2.1, we set

$$
\varphi(r):=\inf _{u \in \Phi^{-1}(]-\infty, r[)} \frac{\left(\sup _{v \in \Phi^{-1}(]-\infty, r[)} \Psi(v)\right)-\Psi(u)}{r-\Phi(u)} .
$$

Note that $\Phi(0)=0$, and by $\left(i_{1}\right), \Psi(0) \geq 0$. Therefore, for every $r>0$,

$$
\begin{aligned}
\varphi(r) & =\inf _{u \in \Phi^{-1}(]-\infty, r[)} \frac{\left(\sup _{v \in \Phi^{-1}(]-\infty, r[)} \Psi(v)\right)-\Psi(u)}{r-\Phi(u)} \\
& \leq \frac{\sup _{\Phi^{-1}(]-\infty, r[)} \Psi}{r} \\
& =\frac{\sup _{\Phi(u)<r} \int_{\Omega} F(x, u) d x}{r}
\end{aligned}
$$

In view of (1.7) and (2.5), we have 


$$
\begin{aligned}
\Phi^{-1}(]-\infty, r[) & :=\{u \in X: \Phi(u)<r\} \\
& \subseteq\left\{u \in X: \frac{M^{-}}{p}\|u\|^{p}<r\right\} \\
& \subseteq\left\{u \in X:|u(x)|<\left(\frac{K p r}{M^{-}}\right)^{\frac{1}{p}}, \forall x \in \Omega\right\}
\end{aligned}
$$

Let $\left(\sigma_{n}\right)$ be a sequence of positive numbers such that $\left(\sigma_{n}\right) \rightarrow+\infty$ and

$$
\lim _{n \rightarrow+\infty} \frac{\int_{\Omega} \sup _{|t| \leq \sigma_{n}} F(x, t) d x}{\sigma_{n}^{p}}=\alpha<+\infty .
$$

Put $r_{n}:=\frac{M^{-} \sigma_{n}^{p}}{K p}$. From (2.7) and (2.8), we obtain

$$
\begin{aligned}
\varphi\left(r_{n}\right) & \leq \frac{K p}{M^{-}} \frac{\sup _{\left\{u \in X:|u(x)|<\sigma_{n}, \forall x \in \Omega\right\}} \int_{\Omega} F(x, u) d x}{\sigma_{n}^{p}} \\
& \leq \frac{K p}{M^{-}} \frac{\int_{\Omega} \sup _{|t|<\sigma_{n}} F(x, t) d x}{\sigma_{n}^{p}} .
\end{aligned}
$$

Let $\gamma:=\liminf \varphi(r)$. It follows from (2.9) and (2.10) that

$$
\begin{aligned}
\gamma & \leq \liminf _{r \rightarrow+\infty} \varphi\left(r_{n}\right) \\
& \leq \frac{K p}{M^{-}} \lim _{n \rightarrow+\infty} \frac{\int_{\Omega} \sup _{|t| \leq \sigma_{n}} F(x, t) d x}{\sigma_{n}^{p}} \\
& \leq \frac{K p \alpha}{M^{-}}<+\infty .
\end{aligned}
$$

From (2.11), it is clear that $\Lambda \subseteq] 0, \frac{1}{\gamma}[$.

For $\lambda \in \Lambda$, we claim that the functional $I_{\lambda}$ is unbounded from below. Indeed, since 


$$
\frac{1}{\lambda}<\frac{K p L \beta}{M^{-}}
$$

we can consider a consequence $\left(\tau_{n}\right)$ of positive numbers and $\eta>0$ such that $\tau_{n} \rightarrow+\infty$ and

$$
\frac{1}{\lambda}<\eta<\frac{K p L}{M^{-}} \frac{\int_{B\left(x^{0}, \frac{s_{1}}{2}\right)} F\left(x, \tau_{n}\right) d x}{\tau_{n}^{p}},
$$

for $n$ large enough. Define a consequence $\left(w_{n}\right)$ as follow

$$
w_{n}(x)= \begin{cases}0 & x \in \bar{\Omega} \backslash B\left(x^{0}, s_{1}\right) \\ \tau_{n}\left(\frac{4}{s_{1}^{3}} l^{3}-\frac{12}{s_{1}^{2}} l^{2}+\frac{9}{s_{1}} l-1\right) & x \in B\left(x^{0}, s_{1}\right) \backslash B\left(x^{0}, \frac{s_{1}}{2}\right) \\ \tau_{n} & x \in B\left(x^{0}, s_{1}\right) .\end{cases}
$$

We have

$$
\frac{\partial w_{n}(x)}{\partial x_{i}}= \begin{cases}0 & x \in \bar{\Omega} \backslash B\left(x^{0}, s_{1}\right) \cup B\left(x^{0}, \frac{s_{1}}{2}\right) \\ \tau_{n}\left(\frac{12 l\left(x_{i}-x_{i}^{0}\right)}{s_{1}^{3}}-\frac{24\left(x_{i}-x_{i}^{0}\right)}{s_{1}^{2}}+\frac{9\left(x_{i}-x_{i}^{0}\right)}{s_{1} l}-1\right) & x \in B\left(x^{0}, s_{1}\right) \backslash B\left(x^{0}, \frac{s_{1}}{2}\right),\end{cases}
$$

and

$$
\frac{\partial^{2} w_{n}(x)}{\partial x_{i}^{2}}=\left\{\begin{array}{lr}
0 & x \in \bar{\Omega} \backslash B\left(x^{0}, s_{1}\right) \cup B\left(x^{0}, \frac{s_{1}}{2}\right) \\
\tau_{n}\left(\frac{12\left[\left(x_{i}-x_{i}^{0}\right)^{2}+l^{2}\right]}{s_{1}^{3} l}-\frac{24}{s_{1}^{2}}+\frac{9\left[l^{2}-\left(x_{i}-x_{i}^{0}\right)^{2}\right]}{s_{1} l^{3}}\right) & x \in B\left(x^{0}, s_{1}\right) \backslash B\left(x^{0}, \frac{s_{1}}{2}\right) .
\end{array}\right.
$$

According to notations in above, we see that

$$
\int_{\Omega}\left|\Delta w_{n}\right|^{p} d x=\theta_{1} \tau_{n}^{p}, \quad \int_{\Omega}\left|\nabla w_{n}\right|^{p} d x=\theta_{2} \tau_{n}^{p}, \quad \int_{\Omega}\left|w_{n}\right|^{p} d x=\theta_{3} \tau_{n}^{p}
$$

Therefore 


$$
\begin{aligned}
\Phi\left(w_{n}\right) & =\frac{1}{p} \int_{\Omega}\left|\Delta w_{n}\right|^{p} d x+\frac{1}{p} \widehat{M} \quad\left(\int_{\Omega}\left|\nabla w_{n}\right|^{p} d x\right)+\frac{\rho}{p} \int_{\Omega}\left|w_{n}\right|^{p} d x \\
& =\frac{1}{p} \theta_{1} \tau_{n}^{p}+\frac{1}{p} \widehat{M}\left(\theta_{2} \tau_{n}^{p}\right)+\frac{\rho}{p} \theta_{3} \tau_{n}^{p}
\end{aligned}
$$

$$
\begin{aligned}
& \leq \frac{1}{p}\left(\theta_{n} \tau_{n}^{p}+m_{1}^{p-1} \theta_{2} \tau_{n}^{p}+\rho \theta_{3} \tau_{n}^{p}\right) \\
& =\frac{M^{-} \tau_{n}^{p}}{K p L} .
\end{aligned}
$$

On other hand, by $\left(i_{1}\right)$, we have

$$
\begin{aligned}
\Psi\left(w_{n}\right) & =\int_{\Omega} F\left(x, w_{n}\right) d x \\
& \geq \int_{B\left(x^{0}, \frac{s_{1}}{2}\right)} F\left(x, w_{n}\right) d x .
\end{aligned}
$$

So, it follows from (2.12), (2.15) and (2.16) that

$$
\begin{aligned}
I_{\lambda}\left(w_{n}\right) & =\Phi\left(w_{n}\right)-\lambda \Psi\left(w_{n}\right) \\
& \leq \frac{M^{-} \tau_{n}^{p}}{K p L}-\lambda \int_{B\left(x^{0}, \frac{S_{1}}{2}\right)} F\left(x, w_{n}\right) d x \\
& <\frac{M^{-} \tau_{n}^{p}}{K p L}(1-\lambda \eta),
\end{aligned}
$$

for $n$ large enough, so $\lim _{n \rightarrow+\infty} I_{\lambda}\left(w_{n}\right)=-\infty$, and hence the claim follows.

The alternative of Theorem 2.1 case (b) assures the existence of unbounded sequence $\left(u_{n}\right)$ of critical points of the functional $I_{\lambda}$ and the proof of Theorem 1.1 is complete.

\subsection{Proof of Theorem 1.2.}

We take $\Phi$ and $\Psi$ be as before. First, note that

$$
\min _{X} \Phi=\Phi(0)=0 .
$$


Let $\left(\sigma_{n}\right)$ be a sequence of positive numbers such that $\sigma_{n} \rightarrow 0^{+}$and

$$
\lim _{n \rightarrow+\infty} \frac{\int_{\Omega} \sup _{|t| \leq \sigma_{n}} F(x, t) d x}{\sigma_{n}^{p}}=\sigma^{0}<+\infty .
$$

Put

$$
r_{n}:=\frac{M^{-} \sigma_{n}^{p}}{K p} \quad \text { and } \quad \delta:=\liminf _{r \rightarrow 0^{+}} \varphi(r)
$$

Similarly as above, we have

$$
\varphi\left(r_{n}\right) \leq \frac{K p}{M^{-}} \frac{\int_{\Omega} \sup _{|t|<\sigma_{n}} F(x, t) d x}{\sigma_{n}^{p}} .
$$

It follows from (2.19) and (2.20) that

$$
\begin{aligned}
\delta & \leq \liminf _{n \rightarrow+\infty} \varphi\left(r_{n}\right) \\
& \leq \frac{K p \alpha^{0}}{M^{-}}<+\infty .
\end{aligned}
$$

By (2.21), we see that $\left.\Lambda^{0} \subseteq\right] 0, \frac{1}{\delta}\left[\right.$. Now, for $\lambda \in \Lambda^{0}$, we claim that $I_{\lambda}$ has not a local minimum at zero.

Indeed, since $\frac{1}{\lambda}<\frac{K p L \beta^{0}}{M^{-}}$, we can consider a sequence $\left(\tau_{n}\right)$ of positive numbers and $\eta>0$ such that

$\tau_{n} \rightarrow 0^{+}$and

$$
\frac{1}{\lambda}<\eta<\frac{K p L}{M^{-}} \frac{\int_{\left(x^{0}, \frac{s_{1}}{2}\right)} F\left(x, \tau_{n}\right) d x}{\tau_{n}^{p}},
$$

for $n$ large enough. Let $\left(w_{n}\right)$ be the sequence defined in (2.13). By (2.15), (2.16) and (2.22), we obtain 


$$
\begin{aligned}
I_{\lambda}\left(w_{n}\right) & \leq \frac{1}{p} \theta_{1} \tau_{n}^{p}+\frac{1}{p} \widehat{M}\left(\theta_{2} \tau_{n}^{p}\right)+\frac{\rho}{p} \theta_{3} \tau_{n}^{p}-\lambda \int_{B\left(x^{0}, \frac{s_{1}}{2}\right)} F\left(x, w_{n}\right) d x \\
& <\frac{M^{-} \tau_{n}^{p}}{K p L}(1-\lambda \eta) \\
& <0=I_{\lambda}(0),
\end{aligned}
$$

for $n$ large enough. This together with the fact that $\left\|w_{n}\right\| \rightarrow 0$ shows that $I_{\lambda}$ has not a local minimum at zero, and the claim follows.

The alternative of Theorem 2.1 case $(c)$ ensures the existence of sequence $\left(u_{n}\right)$ of pairwise distinct critical points (local minima) of $I_{\lambda}$ such that $\left\|u_{n}\right\| \rightarrow 0$. This completes the proof of Theorem 1.2.

Corollary 2.1. Assume that assumption $\left(i_{1}\right)$ in Theorem 1.1 and $(M)$ hold. Suppose that

$\left(i_{4}\right)$ There exist $x^{0} \in \Omega, s_{1}>0$ as considered in (1.8) such that

$$
\liminf _{\sigma \rightarrow+\infty} \frac{\int_{\Omega} \sup _{|t| \leq \sigma} F(x, t) d x}{\sigma^{p}}<\frac{M^{-}}{K p}<L \limsup _{t \rightarrow+\infty} \frac{\int_{B\left(x^{0}, \frac{s_{1}}{2}\right)} F(x, t) d x}{t^{p}} .
$$

Then, the problem

$$
\begin{cases}\Delta\left(|\Delta u|^{p-2} \Delta u\right)-\left[M\left(\int_{\Omega}|\nabla u|^{p} d x\right)\right]^{p-1} \Delta_{p} u+\rho|u|^{p-2} u=f(x, u) & \text { in } \Omega \\ u=\Delta u=0 & \text { on } \partial \Omega,\end{cases}
$$

admits an unbounded sequence of weak solutions.

Corollary 2.1. Let $f: \mathbb{R} \rightarrow \mathbb{R}$ be a continuous function. Assume that (M) holds and

$\left(i_{5}\right) F(t)=\int_{0}^{t} f(s) d s \geq 0$ for every $t \in[0,+\infty)$

$\left(i_{6}\right)$ There exist $x^{0} \in \Omega, s_{1}>0$ as considered in (1.8) such that 


$$
\liminf _{\sigma \rightarrow+\infty} \frac{\sup _{|t| \leq \sigma} F(t)}{\sigma^{p}}<L\left(\frac{s_{1}}{2}\right)^{N} \frac{\pi^{\frac{N}{2}}}{|\Omega| \Gamma\left(1+\frac{N}{2}\right)} \limsup _{t \rightarrow+\infty} \frac{F(t)}{t^{p}}
$$

Then, for every $\left.\lambda \in \frac{M^{-}}{K p}\right] \frac{\Gamma\left(1+\frac{N}{2}\right)}{L\left(\frac{s_{1}}{2}\right)^{N} \pi^{\frac{N}{2}} \limsup _{t \rightarrow+\infty} \frac{F(t)}{t^{p}}}, \frac{1}{|\Omega| \liminf _{\sigma \rightarrow+\infty} \frac{\sup _{|t| \leq \sigma} F(t)}{\sigma^{p}}}[$, the problem

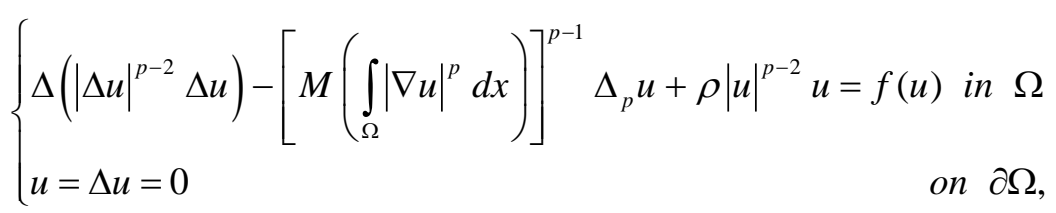

admits an unbounded sequence of weak solutions.

Proof. We set $F(x, t)=F(t)$ for all $x \in \Omega$ and $t \in \mathbb{R}$. Since

$$
\left|B\left(x^{0}, \frac{s_{1}}{2}\right)\right|=\left(\frac{s_{1}}{2}\right)^{N} \frac{\pi^{\frac{N}{2}}}{\Gamma\left(1+\frac{N}{2}\right)}
$$

the result follows from Theorem 1.1.

\section{A remarkable consequences}

This section is concerned, as consequence of Theorem 1.1, with the existence of infinitely many solutions for the following problem

$$
\begin{cases}\Delta\left(|\Delta u|^{p-2} \Delta u\right)-\left[M\left(\int_{\Omega}|\nabla u|^{p} d x\right)\right]^{p-1} \Delta_{p} u+\rho|u|^{p-2} u=\lambda g(x, u)+\mu h(x, u) & \text { in } \Omega \\ u=\Delta u=0 & \text { on } \partial \Omega,\end{cases}
$$


where $p>\max \left\{1, \frac{N}{2}\right\}, \Omega \subset \mathbb{R}^{N}(N \geq 1)$ is a bounded smooth domain, $\lambda, \rho>0, \mu \geq 0, g: \bar{\Omega} \times \mathbb{R} \rightarrow \mathbb{R}$ is a continuous function, $h: \Omega \times \mathbb{R} \rightarrow \mathbb{R}$ is a function such that $h(., t)$ is measurable in $\Omega$ for all $t \in \mathbb{R}$ and $h(x,$.$) is continuous in \mathbb{R}$ for a.e. $x \in \Omega$ and $M: \mathbb{R}^{+} \rightarrow \mathbb{R}$ is a continuous function, which satisfies the condition (M).

Let us now state our main results of this section.

Theorem 3.1. Assume that (M) holds and

(i $\left.i_{7}\right) G(x, t):=\int_{0}^{t} g(x, s) d s \geq 0$ for every $(x, t) \in \Omega \times[0,+\infty)$;

$\left(i_{8}\right)$ There exist $x^{0} \in \Omega, s_{1}>0$ as considered in (1.8) such that, if we put

$$
\alpha^{\prime}:=\liminf _{\sigma \rightarrow+\infty} \frac{\int_{\Omega} \sup _{|t| \leq \sigma} G(x, t) d x}{\sigma^{p}}, \quad \beta^{\prime}:=\limsup _{t \rightarrow+\infty} \frac{\int_{\left(x^{0}, \frac{s_{1}}{2}\right)} G(x, t) d x}{t^{p}},
$$

one has

$$
\alpha^{\prime}<L \beta^{\prime} .
$$

Then, for every

$$
\left.\lambda \in \Lambda^{\prime}:=\frac{M^{-}}{K p}\right] \frac{1}{L \beta^{\prime}}, \frac{1}{\alpha^{\prime}}[
$$

for every function $h: \Omega \times \mathbb{R} \rightarrow \mathbb{R}$ measurable in $\Omega$ and continuous in $\mathbb{R}$ with $H(x, t)=\int_{0}^{t} h(x, s) d s \geq 0$ for all $(x, t) \in \Omega \times \mathbb{R}$, satisfying the conditions

$$
\begin{gathered}
\sup _{|t| \leq \sigma}|h(., t)| \in L^{1}(\Omega), \text { for all } \sigma>0, \\
H_{\infty}:=\lim _{\sigma \rightarrow+\infty} \frac{\int_{\Omega} \sup _{|t| \leq \sigma} H(x, t) d x}{\sigma^{p}}<\infty,
\end{gathered}
$$


and for every $\mu \in\left[0, \mu_{h, \lambda}[\right.$ where

$$
\mu_{h, \mu}:= \begin{cases}\frac{M^{-}}{K p H_{\infty}}\left(1-\lambda \frac{K p \alpha^{\prime}}{M^{-}}\right) & \text {if } H_{\infty} \neq 0 \\ +\infty & \text { if } H_{\infty}=0\end{cases}
$$

problem (3.1) admits an unbounded sequence of weak solutions.

Proof. Our aim is to apply Theorem 1.1. To this end, fix $\lambda \in \Lambda^{\prime}$. Then $1-\lambda \frac{K p \alpha^{\prime}}{M^{-}}>0$, and so $\mu_{h, \lambda}>0$.

Let $\mu \in\left[0, \mu_{h, \lambda}\left[\right.\right.$. If $\mu=0$, the theorem gives back to Theorem 1.1. Let us assume that $0<\mu<\mu_{h, \lambda}$ and put $\lambda_{1}:=\frac{M^{-}}{K p L \beta^{\prime}}$ and $\lambda_{2}:=\frac{\frac{M^{-}}{K p}}{\alpha^{\prime}+\frac{\mu}{\lambda} H_{\infty}}$.

If $H_{\infty}=0$, clearly we have $\left.\lambda \in\right] \lambda_{1}, \lambda_{2}[$.

If $H_{\infty} \neq 0$, it follows from $\mu<\mu_{h, \lambda}$ that $\mu \frac{K p H_{\infty}}{M^{-}}+\lambda \frac{K p \alpha^{\prime}}{M^{-}}<1$, that is $\lambda<\frac{\frac{M^{-}}{K p}}{\alpha^{\prime}+\frac{\mu}{\lambda} H_{\infty}}$, and so $\lambda<\lambda_{2}$.

On other hand, by our hypothesis we have $\lambda>\lambda_{1}$. Hence $\left.\lambda \in\right] \lambda_{1}, \lambda_{2}[$. Now set

$$
F(x, t):=G(x, t)+\frac{\mu}{\lambda} H(x, t), \text { for all } x \in \Omega \text { and } t \in \mathbb{R} \text {. }
$$

Then

$$
\begin{aligned}
\frac{\int_{\Omega} \sup _{|t| \leq \sigma} F(x, t) d x}{\sigma^{p}} & =\frac{\int_{\Omega} \sup _{|t| \leq \sigma}\left[G(x, t)+\frac{\mu}{\lambda} H(x, t)\right] d x}{\sigma^{p}} \\
& \leq \frac{\int_{\Omega} \sup _{|t| \leq \sigma} G(x, t) d x}{\sigma^{p}}+\frac{\mu}{\lambda} \frac{\int_{\Omega} \sup _{|t| \leq \sigma} H(x, t) d x}{\sigma^{p}},
\end{aligned}
$$

and from (3.4), we deduce 


$$
\begin{aligned}
\alpha & :=\liminf _{\sigma \rightarrow+\infty} \frac{\int_{\Omega} \sup _{|t| \leq \sigma} F(x, t) d x}{\sigma^{p}} \\
& \leq \liminf _{\sigma \rightarrow+\infty} \frac{\int_{\Omega} \sup _{|t| \leq \sigma} G(x, t) d x}{\sigma^{p}}+\frac{\mu}{\lambda} H_{\infty} \\
& =\alpha^{\prime}+\frac{\mu}{\lambda} H_{\infty} .
\end{aligned}
$$

Moreover, since $H$ is nonnegative, we see that

$$
\begin{aligned}
\beta & :=\limsup _{t \rightarrow+\infty} \frac{\int_{B\left(x^{0}, \frac{s_{1}}{2}\right)} F(x, t) d x}{t^{p}} G(x, t) d x \\
& \geq \limsup _{t \rightarrow+\infty} \frac{\int_{\left(x^{0}, \frac{s_{1}}{2}\right)}}{t^{p}} \\
& =\beta^{\prime} .
\end{aligned}
$$

Therefore (3.5) and (3.6) yield

$$
\left.\frac{M^{-}}{K p}\right] \frac{1}{L \beta^{\prime}}, \frac{1}{\alpha^{\prime}+\frac{\mu}{\lambda} H_{\infty}}\left[\subseteq \frac{M^{-}}{K p}\right] \frac{1}{L \beta}, \frac{1}{\alpha}[
$$

and hence

$$
\lambda \in] \lambda_{1}, \lambda_{2}\left[\subseteq \Lambda:=\frac{M^{-}}{K p}\right] \frac{1}{L \beta}, \frac{1}{\alpha}[
$$

From (3.7), we observe that condition (1.9) is verified. Consequently, the conclusion follows by applying Theorem 1.1.

Remark 3.1. If

$$
\liminf _{\sigma \rightarrow+\infty} \frac{\int_{\Omega} \sup _{|t| \leq \sigma} G(x, t) d x}{\sigma^{p}}=0 \text { and } \limsup _{t \rightarrow+\infty} \frac{\int_{B\left(x^{0}, \frac{s_{1}}{2}\right)} G(x, t) d x}{t^{p}}=+\infty \text {, }
$$


clearly condition (3.2) is verified and Theorem 3.1 ensures the existence of infinitely many weak

solutions for problem (3.1), for every $(\lambda, \mu) \in] 0,+\infty\left[\times\left[0, \frac{M^{-}}{\mathrm{KpH}_{\infty}}[\right.\right.$. Moreover, under the condition

$H_{\infty}=0$ problem (3.1) admits infinitely many solutions, for every $\left.(\lambda, \mu) \in\right] 0,+\infty[\times[0,+\infty[$.

In view of Theorem 1.2, arguing as in the proof of Theorem 3.1, we have the following result.

Theorem 3.2. Assume that (M), $\left(i_{7}\right)$ hold and

$\left(i_{9}\right)$ There exist $x^{0} \in \Omega, s_{1}>0$ as considered in (1.8) such that, if we put

$$
\alpha^{0^{\prime}}:=\liminf _{\sigma \rightarrow 0^{+}} \frac{\int_{\Omega} \sup _{|t| \leq \sigma} G(x, t) d x}{\sigma^{p}}, \quad \beta^{0^{\prime}}:=\limsup _{t \rightarrow 0^{+}} \frac{\int_{\left(x^{0}, \frac{s_{1}}{2}\right)} G(x, t) d x}{t^{p}},
$$

one has

$$
\alpha^{0^{\prime}}<L \beta^{0^{\prime}}
$$

Then, for every

$$
\left.\lambda \in \Lambda^{0^{\prime}}:=\frac{M^{-}}{K p}\right] \frac{1}{L \beta^{0^{\prime}}}, \frac{1}{\alpha^{0^{\prime}}}[,
$$

for every function $h: \Omega \times \mathbb{R} \rightarrow \mathbb{R}$ measurable in $\Omega$ and continuous in $\square$ with $H(x, t)=\int_{0}^{t} h(x, s) d s \geq 0$ for all $(x, t) \in \Omega \times \mathbb{R}$, satisfying the conditions

$$
\begin{gathered}
\sup _{|t| \leq \sigma}|h(., t)| \in L^{1}(\Omega), \text { for all } \sigma>0, \\
H_{0}:=\lim _{\sigma \rightarrow 0^{+}} \frac{\int_{\Omega} \sup _{|t| \leq \sigma} H(x, t) d x}{\sigma^{p}}<\infty,
\end{gathered}
$$

and for every $\mu \in\left[0, \mu_{h, \lambda}[\right.$ where 


$$
\mu_{h, \mu}:= \begin{cases}\frac{M^{-}}{K p H_{0}}\left(1-\lambda \frac{K p \alpha^{0^{\prime}}}{M^{-}}\right) & \text {if } H_{0} \neq 0 \\ +\infty & \text { if } H_{0}=0,\end{cases}
$$

problem (3.1) admits a sequence $\left(u_{n}\right)$ of weak solutions such that $u_{n} \rightarrow 0$ strongly.

\section{ACKNOWLEDGEMENTS}

The authors are very thankful for the many helpful suggestions and corrections given by the referees who reviewed this paper.

\section{References}

[1] G. A. Afrouzi, S. Heidarkhani, D. O'Regan, Existence of three solutions for doubly eigenvalue fourthorder boundary value problem, Taiwanese J. Math. 15, No. 1 (2011) 201-210.

[2] G. A. Afrouzi, S. Shamilo, M. Mahdavi, Three solution for a class of quasilinear Dirichlet elliptic systems involving $(p, q)$-Laplcian operator, The Journal of mathematics and computer Science. Vol. 4 No. 3 (2012) 487-493.

[3] C. O. Alves, F. J. S. A. Corrêa, T. F. MA, Positive solutions for a quasilinear elliptic equation of Kirchhoff type, Comput. Math. Appl, 49 (2005) 85-93.

[4] G. Bonanno, B. Di Bella, A Boundary value problem for fourth-order elastic beam equations, J. Math. Anal. Appl. 343 (2008) 1166-1176.

[5] G. Bonanno, B. Di Bella, A fourth-order boundary value for a strum-Liouville type equation, Appl. Math. Comput. 217 (2010) 3635-3640.

[6] G. Bonanno, B. Di Bella, D. O'Regan, Non-trivial solutions for nonlinear fourth-order elastic beam equations, Computers and Mathematics with applications 62 (2011) 1862-1869.

[7] G. Bonanno, G. Molica Bisci, Infinitely many solutions for a boundary value problem with discontinuous nonlinearities, Bound. Value Probl. (2009) 1-20.

[8] P. Candito, L. Li, R. Livrea, Infintely many solution for a perturbed nonlinear Navier boundary value problem involving the p-biharmonic, Nonlinear Analysis, 75 (2012) 6360-6369.

[9] P. Candito, R. Livrea , Infintely many solution for a nonlinear Navier boundary value problem involving p-biharmonic, Studia Univ."Babes-Bolyai", Mathematica, 4 (2010).

[10] B. Cheng, X. Wu, J. Liu, Multiplicity of Solutions for Nonlocal Elliptic System of (p,q)-Kirchhoff Type, Abstract and Applied Analysis, Volume 2011, Article ID 526026, 13 pages doi:10.1155/2011/526026.

[11] A. Hamydy, M. Massar, N. Tsouli, Existence of solutions for p-Kirchhoff type problems with critical exponent, Electron. J. Diff. Equ. Vol. 2011 (2011), No. 105, pp. 1-8. 
[12] S. Heidarkhani, G. A. Afrouzi, Multiplicity results for a Kirchhoff type doubly eigenvalue boundary value problem, The Journal of mathematics and computer Science. Vol. 3 No. 1 (2011) 11-20.

[13] X. He, W. Zou, Infinitely many positive solutions for Kirchhoff-type problems, Nonlinear Analysis Theory, Methods and Applications, 70, 3, (2009) 1407-1414.

[14] E. M. Hssini, M. Massar, M. Talbi, N. Tsouli, Infinitely Many Solutions for Nonlocal Elliptic pKirchhoff Type Equation under Neumann Boundary Condition, Int. Journal of Math. Analysis, Vol. 7, (2013), no. 21, 1011-1022.

[15] G. Kirchhoff, Mechanik, Teubner, leipzig, Germany, 1883.

[16] M. Massar, Existence and multiplicity solutions for nonlocal elliptic problems, Electron. J. Diff. Equ. 2013 (2013), No. 75, pp. 1-14.

[17] M. Massar, El. Hssini, N. Tsouli, Infinitely many solutions for class of Navier boundary (p,q)biharmonic systems, Electron. J. Diff. Equ. Vol. 2012 (2012) No. 163, pp. 1-9.

[18] M. K. Moghadam, G.A. Afrouzi, J. Vahidi, A minimax inequality for a class of functional and its applications to existence of multiple solutions for elliptic equation, The Journal of mathematics and computer Science. Vol. 4 No. 3 (2012) 350-360.

[19] B. Ricceri, A general variational principle and some of its applications, J. Comput. Appl. Math. 133 (2000) 401-410.

[20] Y. Shen, J. Zhang, Multiplicity of positive solutions for a Navier boundary-value problem involving the p-biharmonic with critical exponent, Electronic J. of Differential Equations, 47 (2011) 1-14.

[21] F. Wang, Y. An, Existence and multiplicity of solutions for a fourth-order elliptic equation, Boundary Value Problems 2012, 2012:6. 